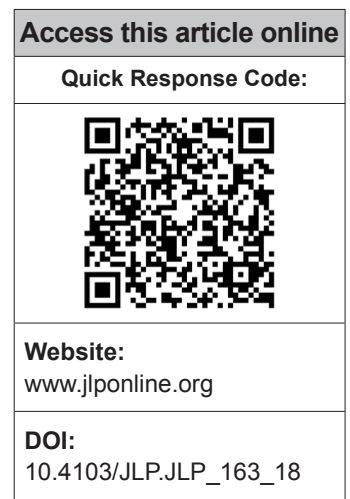

Departments of ${ }^{1}$ Biochemistry and ${ }^{2}$ Community and Family Medicine, All India Institute of Medical Sciences, Rishikesh, Uttarakhand, ${ }^{3}$ Department of Biochemistry, Lady Hardinge Medical College, New Delhi, India

Address for correspondence: Dr. Rajeev Goyal, Department of Biochemistry, Lady Hardinge Medical College, New Delhi - 110054 , India.

E-mail: docrajeev@gmail. com

Submission: 03-12-2018 Accepted: 11-06-2019

\title{
Single point insulin sensitivity estimator as an index for insulin sensitivity for metabolic syndrome: A study in North Indian population
}

\author{
Parmila Dudi', Bela Goyal', Vartika Saxena ${ }^{2}$, Kamlesh Rabari ${ }^{1}$, Anissa Atif Mirza ${ }^{1}$, \\ Manisha Naithani ${ }^{1}$, Tarun Kumar ${ }^{1}$, Rajeev Goyal ${ }^{1,3}$
}

\begin{abstract}
:
BACKGROUND: Various indices for estimating insulin sensitivity, based on glucose tolerance test and fasting insulin levels, have been devised. However, they are laborious, time-consuming, and costly. Recently, a new index, single point insulin sensitivity estimator (SPISE) based on TG, high-density lipoproteins (HDL), and body mass index (BMI) was proposed in the European population and was found comparable to gold standard test. Decreased insulin sensitivity is a hallmark of metabolic syndrome (MetS). Hence, the current study was planned to determine the optimal cutoff of SPISE with high sensitivity and specificity in MetS patients of the North Indian population.
\end{abstract}

MATERIALS AND METHODS: A community-based cross-sectional study including 229 MetS cases and 248 controls was conducted. MetS was defined according to the South Asian Modified National Cholesterol Education Program criteria. SPISE index was calculated for cases and controls using the formula devised by Paulmichl et al:: SPISE $=600 \times \mathrm{HDL}-\mathrm{C}^{0.185} /\left(\mathrm{TG}^{0.2} \times \mathrm{BMI}^{1.338}\right)$. Receiver operating characteristic (ROC) curve was plotted for determining optimal cutoff for SPISE in MetS.

RESULTS: SPISE was significantly lower in MetS patients $(5.35 \pm 1.35)$ than that for controls $(7.45 \pm 2)$ with $P<0.05$ (confidence interval [CI]: 1.79-2.41). ROC curve showed area under the curve $=0.83$ for SPISE $(P<0.05, \mathrm{Cl}$ : $0.79-0.86)$, showing SPISE to have good predictive ability to discriminate MetS cases from controls. The cutoff value of SPISE index for predicting insulin sensitivity in MetS was found out to be 5.82 with sensitivity and specificity of $73 \%$ and $80 \%$, respectively. This cutoff is lower than the European population (6.61), indicating higher insulin resistance (IR) in the study population.

CONCLUSION: SPISE could be a useful potential low-cost indicator with high sensitivity and specificity for predicting IR in MetS.

Key words:

Insulin resistance, metabolic syndrome, single point insulin sensitivity estimator

\section{Introduction}

Tetabolic syndrome (MetS) consists of a constellation of metabolic abnormalities that confer increased risk of cardiovascular diseases (CVDs) and type II diabetes. A meta-analysis found that MetS was associated with relative risk of 2.99 and

This is an open access journal, and articles are distributed under the terms of the Creative Commons Attribution-NonCommercial-ShareAlike 4.0 License, which allows others to remix, tweak, and build upon the work non-commercially, as long as appropriate credit is given and the new creations are licensed under the identical terms.

For reprints contact: reprints@medknow.com
1.65 for developing type II diabetes and CVD, respectively..$^{[1]}$ National Health and Nutrition Examination Survey (NHANES) reported the age-adjusted prevalence of MetS between 2009 and 2010 at $22.9 \%$, for United States population. ${ }^{[2]}$ ICMR-INdia DIABetes study (2008-2011) reported a prevalence of $16.7 \%-41.7 \%$ in urban and $8.5 \%-25.8 \%$ in rural areas in India. ${ }^{[3]}$ The prevalence of MetS is on the rise. The chief

\footnotetext{
How to cite this article: Dudi P, Goyal B, Saxena V, Rabari K, Mirza AA, Naithani M, et al. Single point insulin sensitivity estimator as an index for insulin sensitivity for metabolic syndrome: A study in North Indian population. J Lab Physicians 2019;11:244-8.
} 
pathophysiological mechanism of MetS is reported to be resistance to insulin-stimulated glucose uptake, i.e., decreased insulin sensitivity. ${ }^{[4]}$ Various methods are available for measuring insulin sensitivity. Euglycemic hyperinsulinemic clamp test based on glucose infusion is gold standard for the measurement of insulin sensitivity. ${ }^{[5]}$ However, hyperinsulinemic clamp test is very complicated, laborious, uncomfortable for a patient, and expensive. Thus, relatively less labor-intensive and less-expensive indices based on glucose tolerance tests have been developed as surrogate measures of insulin sensitivity such as minimal model sensitivity index, calculated sensitivity index, and Matsuda composite insulin sensitivity index (ISI). ${ }^{[6-8]}$ However, these tests require multiple sampling and hence are inconvenient. Simpler approaches employing single fasting sample, such as homeostasis model assessment-insulin resistance (HOMA-IR) and quantitative insulin sensitivity check index (QUICKI), have been developed, but these indices require insulin estimation, which is a costly parameter and lacks universal availability. Triglycerides/high-density lipoproteins cholesterol ratio (TG/HDL-C) was introduced as an affordable tool for estimating IR with the advantage of universal availability and cost-effectiveness. ${ }^{[11]}$ However, it was shown to lack specificity and showed high variability with respect to different populations. ${ }^{[12]}$ Hence, there is an impending need to develop a less costly, simple, and specific index for the measurement of insulin sensitivity, especially in resource-poor countries like India. Paulmichl et al. refined the TG/HDL-C ratio and introduced an index, single point insulin sensitivity estimator (SPISE) as a promising tool for estimating IR based on single fasting sample for TG, HDL-C, and body mass index (BMI). ${ }^{[13]}$ SPISE was devised using computer-assisted mathematical modeling of their study population data. Multiple mathematical models were compared to $M$-value derived from euglycemic hyperinsulinemic clamp test. Each modeling result was scored by correlation coefficient with $M$-value and identification of IR. The proposed model described as SPISE showed moderate-to-strong positive correlations in the studied population. They also analyzed area under the receiver operating characteristic (ROC) curve for various insulin sensitivity indices and used $M$-value of $<4.7 \mathrm{mg} / \mathrm{kg} / \mathrm{min}$ as the definition of higher IR. ${ }^{[13]}$ SPISE showed comparable results to HOMA-IR and QUICKI and significantly better results than TG/ HDL-C ratio. A cutoff value of 6.61 for SPISE index was proposed for their population, smaller values indicating IR. ${ }^{[13]}$ However, this cutoff was based on the study in the European population by investigating healthy adults and obese adolescents. Indian population belongs to a different ethnicity so much so that National Cholesterol Education Program (NCEP) guidelines defining MetS have been modified for South Asians, whereby the cutoff of waist circumference is lowered..$^{[14]}$ Furthermore, we as a developing country cannot afford expensive investigations for IR. Moreover, MetS patients whose hallmark is IR were not included in Paulmichl et al.'s study. Hence, the current study was planned to determine optimal cutoff of SPISE with high sensitivity and specificity in MetS patients of the North Indian population.

\section{Materials and Methods}

A community-based, observational cross-sectional study was carried out from September 2015 to August 2016 at the Department of Biochemistry, of a tertiary care teaching hospital in North India. Ethical approval for the project was taken from institutional ethical committee and written informed consent was taken from all the participants.

\section{Selection of subjects}

The sample size was calculated by the following formula as described by Hajian-Tilaki ${ }^{[15]}$ and was estimated to be 229:

$n=\frac{Z_{\frac{\alpha}{2}}^{2} S p(1-S p)}{d^{2}(1-p r e v)}$

where " $n$ " is sample size, "Sp" ascertained by previous published data, $80 \%$; ${ }^{[13]}$ " $d$ " is degree of precision, $6 \%$; "prev" is prevalence of MetS, $25 \% ;{ }^{[3]}$ and for $\alpha=0.05$, $Z_{\frac{\alpha}{2}}$ is inserted by 1.96 .

$\overline{2}$

It was a convenient sampling method. Volunteers $>18$ years from AIIMS staff (doctors, nurses, and technical and clerical staff), banks, and schools of Rishikesh were enrolled.

MetS was defined according to the South Asian Modified NCEP criteria where three of the five risk factors are present: enlarged waist circumference $\geq 90 \mathrm{~cm}$ in men and $\geq 80 \mathrm{~cm}$ in women, TG $\geq 150 \mathrm{mg} / \mathrm{dL}$ or drug treatment for elevated TG, HDL-C $<40 \mathrm{mg} / \mathrm{dL}$ in men and $<50 \mathrm{mg} / \mathrm{dL}$ in women, systolic blood pressure (BP) $\geq 130 \mathrm{mmHg}$ or diastolic $\mathrm{BP} \geq 85 \mathrm{mmHg}$ or drug treatment for elevated BP, and fasting glucose $>100 \mathrm{mg} / \mathrm{dL}$ or drug treatment for elevated glucose. ${ }^{[14]}$

Individuals with Cushing syndrome, hypothyroidism, secondary hypertension, type 1 diabetes, hepatobiliary disease, nephritic syndrome, or pregnancy were excluded from the study. Finally, a total of 477 individuals were selected.

\section{Methods}

For each subject, demographics such as age, gender, 
residential address, ethnicity, level of education, and household income and medical history were documented. Furthermore, BP, height, weight, waist circumference, and hip circumference were measured, and BMI was also calculated.

Four milliliters of the venous blood was collected from each subject: $2 \mathrm{ml}$ in a Na-fluoride vacutainer for glucose, and $2 \mathrm{ml}$ in a plain vacutainer for lipid profile.

Fasting blood sugar (FBS), TG, and HDL-C were measured on an AU5800 Clinical Chemistry Automated System (Beckman Coulter, Inc.), by hexokinase, glycerol phosphate oxidase-peroxidase, and enzymatic inhibition method, respectively. Quality control samples provided by the Beckman Coulter were used for routine quality control check on the autoanalyzer. When internal quality check was found to be satisfactory, then only study subject's samples were analyzed.

The cohort was then grouped into MetS $(n=229)$ and non-MetS $(n=248)$.

SPISE index was calculated for both the groups using the following formula: ${ }^{[13]}$

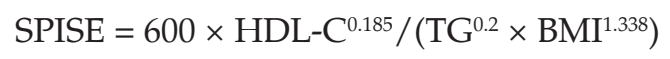

\section{Statistical analyses}

All the statistical analyses were performed using SPSS (v 22; SPSS Inc., Chicago, IL, USA). Data were represented as mean \pm standard deviation. The mean value of SPISE was compared between cases and controls using Student's $t$-test. ROC curve was plotted for area under the curve (AUC) to assess the discriminatory ability of SPISE to differentiate between MetS cases and controls and to determine the optimal cutoff value of SPISE index for MetS. Whole cohort was regrouped into SPISE above and below the cutoff. These groups were then compared for various factors responsible for MetS, viz., waist circumference, BMI, systolic BP, diastolic BP, TG, HDL-C, and FBS by Student's $t$-test. $P<0.05$ was considered statistically significant.

\section{Results}

A total of 477 subjects were investigated in our study, consisting of 229 cases of MetS and 248 controls. The age of the cases ranged from 21 to 78 years and that of controls ranged from 18 to 78 years. Details of data on age, gender, and anthropometric and biochemical parameters of cases and controls are shown in Table 1.

The mean value of SPISE index was found to be significantly lower in MetS patients $(5.35 \pm 1.35)$ than that for controls $(7.45 \pm 2)$ with $P<0.05$ (confidence interval [CI]: 1.79-2.41).
ROC curve between true-positive rate and false-positive rate showed AUC for SPISE index to be $0.83(P<0.05$, CI: 0.79-0.86) [Figure 1].

By studying coordinates of ROC, the cutoff value of SPISE index for predicting insulin sensitivity in MetS was found out to be 5.82 , with sensitivity and specificity of $73 \%$ and $80 \%$, respectively. Further, on grouping individuals on

Table 1: Descriptive characteristics of cases and controls

\begin{tabular}{lc|c}
\hline Characteristic & Cases $(\boldsymbol{n}=\mathbf{2 2 9})$ & Controls $(\boldsymbol{n}=\mathbf{2 4 8})$ \\
\hline Age (years) & $46.91 \pm 12.53$ & $38.38 \pm 14.73$ \\
Gender (male/female) & $127 / 102$ & $192 / 56$ \\
BMI $\left(\mathrm{kg} / \mathrm{m}^{2}\right)$ & $27.21 \pm 4.27$ & $23.3 \pm 4.09$ \\
Waist circumference (inch) & $36.43 \pm 6.63$ & $33.32 \pm 6.54$ \\
Systolic BP (mmHg) & $148 \pm 22$ & $130 \pm 22$ \\
Diastolic BP (mmHg) & $91 \pm 11$ & $82 \pm 13$ \\
TG $(\mathrm{mg} / \mathrm{dL})$ & $194 \pm 119$ & $117 \pm 78$ \\
HDL $(\mathrm{mg} / \mathrm{dL})$ & $43 \pm 11$ & $43 \pm 8$ \\
FBS $(\mathrm{mg} / \mathrm{dL})$ & $111 \pm 38$ & $94 \pm 31$ \\
\hline
\end{tabular}

$\mathrm{BMl}=$ Body mass index, $\mathrm{BP}=\mathrm{Blood}$ pressure, $\mathrm{TG}=$ Triglycerides,

$\mathrm{HDL}=$ High-density lipoproteins, FBS=Fasting blood sugar

Table 2: Comparison between groups formed using single point insulin sensitivity estimator index cutoff 5.82

\begin{tabular}{lccc}
\hline Parameter & $\begin{array}{c}\text { SPISE }<5.82 \\
(\boldsymbol{n}=\mathbf{2 1 6})\end{array}$ & $\begin{array}{c}\text { SPISE } \geq \mathbf{5 . 8 2} \\
(\boldsymbol{n}=\mathbf{2 5 9})\end{array}$ & $P$ \\
\hline BMI $\left(\mathrm{kg} / \mathrm{m}^{2}\right)$ & $28.47 \pm 3.85$ & $22.22 \pm 2.77$ & $<0.05$ \\
Waist circumference (inch) & $36.6 \pm 3.71$ & $33.3 \pm 8.23$ & $<0.05$ \\
Systolic BP $(\mathrm{mmHg})$ & $144 \pm 23$ & $135 \pm 23$ & $<0.05$ \\
Diastolic BP $(\mathrm{mmHg})$ & $93 \pm 54$ & $83 \pm 12$ & $<0.05$ \\
TG $(\mathrm{mg} / \mathrm{dL})$ & $208 \pm 128$ & $109 \pm 53$ & $<0.05$ \\
HDL $(\mathrm{mg} / \mathrm{dL})$ & $39 \pm 7$ & $44 \pm 11$ & $<0.05$ \\
FBS $(\mathrm{mg} / \mathrm{dL})$ & $106 \pm 35$ & $99 \pm 36$ & $<0.05$ \\
\hline
\end{tabular}

SPISE=Single point insulin sensitivity estimator, $\mathrm{BMI}=$ Body mass index, $\mathrm{BP}=\mathrm{Blood}$ pressure, $\mathrm{TG}=$ Triglycerides, $\mathrm{HDL}=$ High-density lipoproteins, FBS=Fasting blood sugar

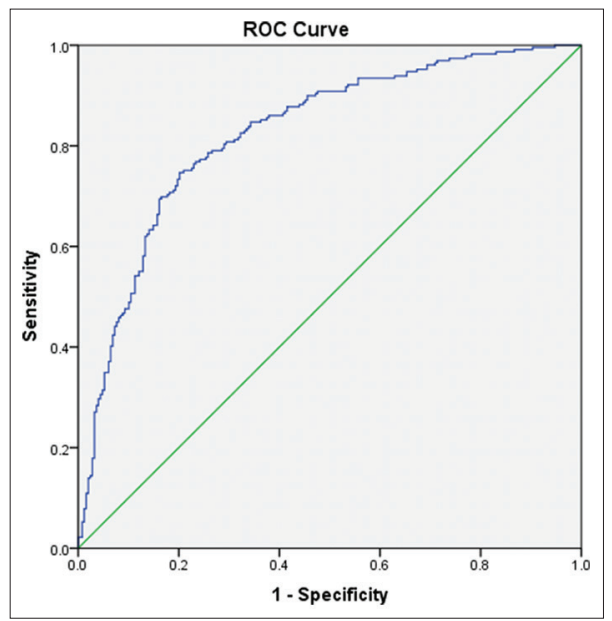

Figure 1: Receiver operating characteristic curve representing area under the curve of single point insulin sensitivity estimator index for differentiating metabolic syndrome cases from controls 
the basis of cutoff value of SPISE 5.82, as described in Table 2, it was found that waist circumference, BMI, systolic BP, diastolic BP, TG, and FBS were significantly higher in SPISE index $<5.82$ group as compared to those in SPISE index $\geq 5.82$ group. However, HDL was significantly lower in SPISE index $<5.82$ group as compared to that in SPISE index $\geq 5.82$ group. This shows that SPISE index 5.82 gives good demarcation between the two groups.

\section{Discussion}

With the rising prevalence of MetS and its known association with increased risk of type II diabetes mellitus and CVD, it is of paramount importance to identify these cases at earliest. Any biomarker/index that can predict IR in MetS early can be a potential aid in assessing the severity of MetS.

Although various indices based on glucose tolerance test and fasting insulin levels have earlier been devised, they are laborious, time-consuming, and costly. ${ }^{[6-10]}$ TG/HDL-C ratio was devised two decades earlier and was proven to be a simple and effective index in predicting IR. ${ }^{[11]}$ However, it showed to lack specificity and showed variable results in different populations. ${ }^{[12]}$ Paulmichl et al. recently devised an index, SPISE, based on TG, HDL-C, and BMI and showed it to be comparable to the gold standard euglycemic hyperinsulinemic clamp test and insulin-based HOMA-IR and QUICKI indices for IR in the European population of healthy adults and obese adolescents. ${ }^{[13]}$ However, there is no such study reported in our population. Hence, we conducted this study on North Indian population where we compared SPISE in patients of MetS from that in controls. An AUC of 0.83 was found by ROC curve, which is statistically significant $(P<0.05, \mathrm{CI}$ : 0.79-0.86), showing SPISE to have good predictive ability to discriminate MetS cases from controls. Further, we evaluated an optimal cutoff at which SPISE shows high sensitivity and specificity. We observed at a cutoff value of 5.82 SPISE showed a sensitivity of $73 \%$ and a specificity of $80 \%$. This cutoff of SPISE is much lower than that of European population where Paulmichl et al. found a SPISE cutoff value of 6.61, at corresponding specificity. This signifies that our population has higher IR, i.e., decreased insulin sensitivity. This is also shown by a previous study conducted by Petersen et al., where they have clearly shown higher IR as well as increased prevalence of IR in the Asian-Indian group (59\%) compared with other ethnic groups (Caucasian [20\%], Eastern-Asian [30\%], Black [33\%], and Hispanic [18\%]). ${ }^{[16]}$ Higher prevalence of IR in India could be ascribed to susceptibility of Indians for developing truncal obesity, intra-abdominal fat and ectopic fat deposition (liver fat), urban habits, and limited physical activity. ${ }^{[17,18]}$ Biologic basis for this has been hypothesized to be a thrifty gene in Indians that helped survival in the past under conditions of nutritional scarcity that has now become detrimental in conditions of plentiful food and sedentary lifestyle. ${ }^{[19]}$

In our study, when we further compared the two groups formed using SPISE equal to 5.82 as cutoff value for diagnosing MetS, we found that waist circumference, BMI, systolic BP, diastolic BP, TG, FBS, and HDL-C showed a significant difference. This further enhances the significance of SPISE as a diagnostic marker for MetS. Hence, our study has shown SPISE to possess high sensitivity and specificity for predicting severity of MetS. This is a very interesting finding as SPISE index can be considered as a potential low cost routinely available simple index, requiring a single sample that can be calculated in most of the laboratories of our country.

However, there are certain limitations to our study which need to be acknowledged. Our study was a cross-sectional having limitations inherent to its design. In addition, our study population is limited to only one region. Moreover, performance of SPISE index in comparison with the gold standard euglycemic hyperinsulinemic test is lacking. Therefore, we propose large-scale studies with population samples involving different regions of India to be conducted and validating SPISE in comparison with gold standard test, as a diagnostic marker of MetS.

\section{Conclusion}

We propose SPISE index as a useful potential low-cost indicator with high sensitivity and specificity for predicting MetS in the Indian set-up, with limited resources and higher prevalence of IR.

\section{Financial support and sponsorship}

We acknowledge financial support from AIIMS, Rishikesh, through intramural project (Reference No. IEC/IM/05/RC/04).

\section{Conflicts of interest}

There are no conflicts of interest.

\section{References}

1. Ford ES. Risks for all-cause mortality, cardiovascular disease, and diabetes associated with the metabolic syndrome: A summary of the evidence. Diabetes Care 2005;28:1769-78.

2. Beltrán-Sánchez $\mathrm{H}$, Harhay MO, Harhay MM, McElligott S. Prevalence and trends of metabolic syndrome in the adult U.S. Population, 1999-2010. J Am Coll Cardiol 2013;62:697-703.

3. Mohan V. ICMR-INdia DIABetes [INDIAB] Study, Phase I Final Report (2008-2011); 2016. p. 40.

4. Aganović I, Dušek T. Pathophysiology of metabolic syndrome. EJIFCC 2007;18:3-6.

5. Tam CS, Xie W, Johnson WD, Cefalu WT, Redman LM, Ravussin E. 
Defining insulin resistance from hyperinsulinemic-euglycemic clamps. Diabetes Care 2012;35:1605-10.

6. Pacini G, Tonolo G, Sambataro M, Maioli M, Ciccarese M, Brocco $\mathrm{E}$, et al. Insulin sensitivity and glucose effectiveness: Minimal model analysis of regular and insulin-modified FSIGT. Am J Physiol 1998;274:E592-9.

7. Tura A, Sbrignadello S, Succurro E, Groop L, Sesti G, Pacini G. An empirical index of insulin sensitivity from short IVGTT: Validation against the minimal model and glucose clamp indices in patients with different clinical characteristics. Diabetologia 2010;53:144-52.

8. Matsuda M, DeFronzo RA. Insulin sensitivity indices obtained from oral glucose tolerance testing: Comparison with the euglycemic insulin clamp. Diabetes Care 1999;22:1462-70.

9. Matthews DR, Hosker JP, Rudenski AS, Naylor BA, Treacher DF, Turner RC. Homeostasis model assessment: Insulin resistance and beta-cell function from fasting plasma glucose and insulin concentrations in man. Diabetologia 1985;28:412-9.

10. Katz A, Nambi SS, Mather K, Baron AD, Follmann DA, Sullivan G, et al. Quantitative insulin sensitivity check index: A simple, accurate method for assessing insulin sensitivity in humans. J Clin Endocrinol Metab 2000;85:2402-10.

11. McLaughlin T, Abbasi F, Cheal K, Chu J, Lamendola C, Reaven G. Use of metabolic markers to identify overweight individuals who are insulin resistant. Ann Intern Med 2003;139:802-9.

12. Sumner AE, Finley KB, Genovese DJ, Criqui MH, Boston RC. Fasting triglyceride and the triglyceride-HDL cholesterol ratio are not markers of insulin resistance in African Americans. Arch Intern Med 2005;165:1395-400.
13. Paulmichl K, Hatunic M, Højlund K, Jotic A, Krebs M, Mitrakou A, et al. Modification and validation of the triglyceride-to-HDL cholesterol ratio as a surrogate of insulin sensitivity in white juveniles and adults without diabetes mellitus: The single point insulin sensitivity estimator (SPISE). Clin Chem 2016;62:1211-9.

14. Alberti KG, Eckel RH, Grundy SM, Zimmet PZ, Cleeman JI, Donato KA, et al. Harmonizing the metabolic syndrome: A joint interim statement of the International Diabetes Federation Task Force on Epidemiology and Prevention; National Heart, Lung, and Blood Institute; American Heart Association; World Heart Federation; International Atherosclerosis Society; and International Association for the study of obesity. Circulation 2009;120:1640-5.

15. Hajian-Tilaki K. Sample size estimation in diagnostic test studies of biomedical informatics. J Biomed Inform 2014;48:193-204.

16. Petersen KF, Dufour S, Feng J, Befroy D, Dziura J, Dalla Man C, et al. Increased prevalence of insulin resistance and nonalcoholic fatty liver disease in Asian-Indian men. Proc Natl Acad Sci U S A 2006;103:18273-7.

17. Chandalia M, Abate N, Garg A, Stray-Gundersen J, Grundy SM. Relationship between generalized and upper body obesity to insulin resistance in Asian Indian men. J Clin Endocrinol Metab 1999;84:2329-35.

18. Misra A, Khurana L. The metabolic syndrome in South Asians: Epidemiology, determinants, and prevention. Metab Syndr Relat Disord 2009;7:497-514.

19. Yajnik CS. The insulin resistance epidemic in India: Fetal origins, later lifestyle, or both? Nutr Rev 2001;59:1-9. 